\title{
Presentation of multiple myeloma mimicking bone metastasis from colon adenocarcinoma: A case report and literature review
}

\author{
HAICHENG ZHANG ${ }^{1 *}$, JURONG LV ${ }^{1}$, CHAOLIANG LV ${ }^{2}$ and HONGMEI ZHANG ${ }^{2 *}$ \\ ${ }^{1}$ Department of Surgery, Jinxiang People's Hospital, Jining, Shandong 272200; \\ ${ }^{2}$ Department of Spine Surgery, Jining No. 1 People's Hospital, Jining, Shandong 272011, P.R. China
}

Received May 5, 2015; Accepted September 10, 2015

DOI: $10.3892 / \mathrm{mco} .2015 .650$

\begin{abstract}
We herein present the case report of a 83-year-old female patient who had undergone right colon resection for adenocarcinoma 2 years earlier, and developed osteolytic lesions of the right femur 6 months ago. A roentgenogram of the right thigh, technetium- $99 \mathrm{~m}$ phosphate bone scintigraphy and combined ${ }^{18} \mathrm{~F}$-fluoro-2-deoxy-D-glucose positron emission tomography/computed tomography imaging were performed, and the results revealed multiple osteolytic lesions in the humerus bilaterally, the right scapula and the right femur. The lesions were suspected to be colon cancer metastases. To improve the quality of life of the patient, palliative surgery was performed. However, the intraoperative biopsy of the focal lesions and immunohistochemical evaluation revealed multiple myeloma (MM). Chemotherapy was administered 2 weeks after surgery and the patient recovered uneventfully. The manifestations of MM and bone metastases are occasionally similar. Although the coexistence of the two diseases is rare, both conditions should be considered in the differential diagnosis of osteolytic lesions.
\end{abstract}

\section{Introduction}

When a patient presents with manifestations of lytic skeletal lesions and a history of adenocarcinoma of the colon, the presumptive diagnosis tends to be metastases from the colon cancer, since the incidence of osteolytic lesions caused by metastasis may range between 0.6 and $7.9 \%(1,2)$. Only a limited number of cases have been reported with the coexistence of colon adenocarcinoma and multiple myeloma (MM) $(3,4)$. However, considering the significant differences in treatment and prognosis between MM and bone metastases,

Correspondence to: Dr Chaoliang Lv, Department of Spine Surgery, Jining No. 1 People's Hospital, 6 Jiankang Road, Jining, Shandong 272011, P.R. China

E-mail: lvchaolianggk@163.com

\section{${ }^{*}$ Contributed equally}

Key words: bone metastasis, multiple myeloma, osteolytic lesion, colon adenocarcinoma, prognosis timely differentiation between the two is crucial. We herein present the case of a patient with a history of colon adenocarcinoma and a clinical manifestation of osteolytic lesions in the femur, who was ultimately diagnosed with MM, following intraoperative biopsy.

\section{Case report}

An 81-year-old woman underwent right colectomy for Dukes' A adenocarcinoma without lymph node metastases (T1N0M0; stage I) 2 years earlier. The patient did not receive chemotherapy or radiotherapy postoperatively. Six months ago, the patient developed progressive pain in the right thigh, without weight loss, abdominal complaints, or diminished appetite. A right thigh roentgenogram was performed, revealing lytic skeletal lesions of the femur, without periosteal reaction (Fig. 1).

Subsequently, the patient was admitted to our hospital with a presumptive diagnosis of metastases from the colon. However, the physical examination revealed no evidence of local recurrence of colon cancer, and the laboratory examinations, such as colon cancer tumor markers, revealed no abnormal findings, apart from mild anemia. Technetium- $99 \mathrm{~m}$ phosphate bone scintigraphy revealed increased uptake in the right femur. One week later, combined ${ }^{18} \mathrm{~F}$-fluoro-2-deoxy-D-glucose positron emission tomography/computed tomography $\left({ }^{18} \mathrm{~F}-\mathrm{FDG}\right.$ PET/CT) imaging was performed to further investigate the possibility of metastatic disease. Variable degrees of ${ }^{18} \mathrm{~F}$-FDG uptake were observed in the humerus bilaterally, the right scapula and the right femur (Fig. 2). During her stay in hospital, the patient suddenly developed gradually worsening pain in the right thigh. An X-ray revealed a pathological fracture. With a suspected diagnosis of metastasis from colon adenocarcinoma with pathological fracture, palliative surgery was performed by curetting the tumor, filling the bone defect with bone cement and fixing with intramedullary nails to improve life quality (Fig. 3). A biopsy of the focal lesion was performed intraoperatively, revealing large clusters of plasma cells and no evidence of metastatic adenocarcinoma of the colon (Fig. 4). Immunohistochemical staining demonstrated that the neoplastic cells strongly expressed CD138 and IgG $\lambda$-type. Serum protein electrophoresis demonstrated an $\mathrm{M}$ band and urine immunoelectrophoresis revealed $\lambda$ chain Bence-Jones proteinuria.

A diagnosis of $\operatorname{Ig} G \lambda$ MM was finally confirmed. Chemotherapy was immediately administered, with 3 courses 


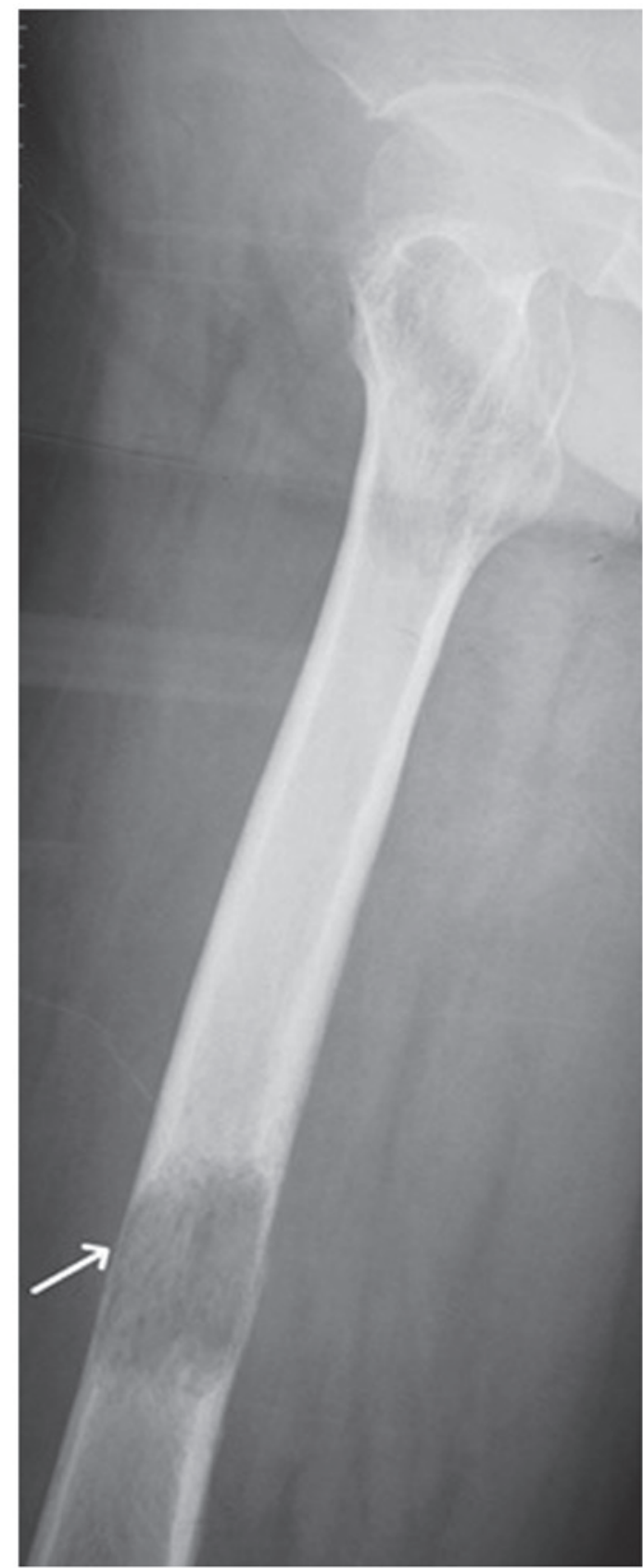

Figure 1. Roentgenogram showing lytic skeletal lesions in the diaphysis of the femur, without periosteal reaction (arrows).

of thalidomide $50 \mathrm{mg} / \mathrm{day}$ and prednisolone $50 \mathrm{mg} / \mathrm{day}$ for 4 consecutive days every 4 weeks. At 3 months postoperatively, the patient had recovered uneventfully, without pain of the affected limbs, had regained her premorbid mobility and was able to tend to herself.

\section{Discussion}

$\mathrm{MM}$ is a malignancy of plasma cells, in which monoclonal antibodies are overproduced. MM accounts for $\sim 10 \%$ of haematological malignancies and exhibits a peak incidence in older men (5). This highly treatable malignancy, mainly presents in the axial skeleton, is characterized by clonal proliferation of plasma cells and usually presents with anemia, bone pains and classical osteolytic lesions without periosteal

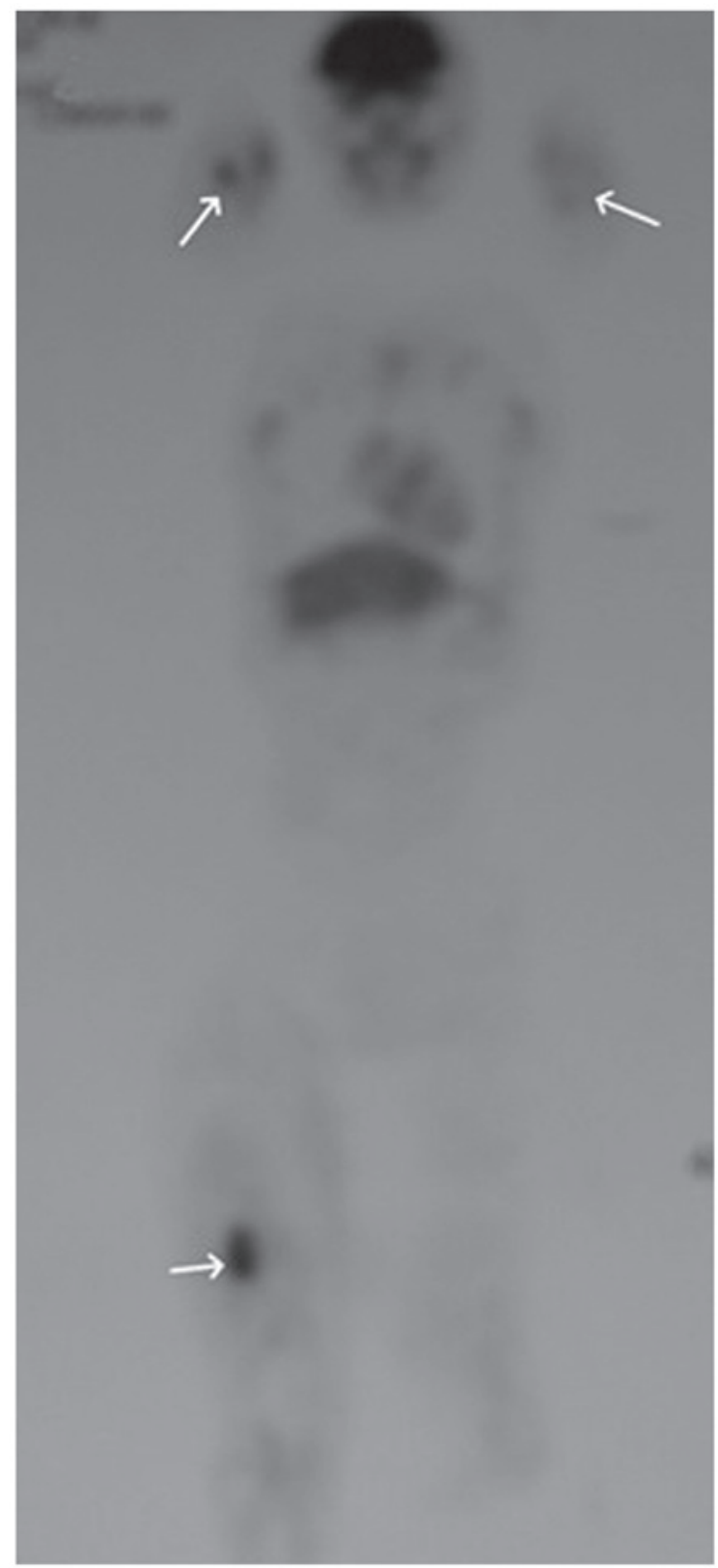

Figure 2. Combined ${ }^{18} \mathrm{~F}$-fluoro-2-deoxy-D-glucose $\left({ }^{18} \mathrm{~F}\right.$-FDG) positron emission tomography/computed tomography imaging revealed several areas with mildly to greatly increased ${ }^{18} \mathrm{~F}$-FDG uptake in the humerus bilaterally, the right scapula and the right femur (arrows).

reaction (6). MM is associated with highly variable clinical manifestations, making its diagnosis very difficult (7). The key laboratory examinations are $\beta 2$-microglobulin, serum protein electrophoresis, serum and urine immunofixation, free light chains, bone marrow aspiration and biopsy with immunophenotyping, conventional cytogenetics and fluorescence in situ hybridization (8-11). The primary modalities of treatment for MM consist of chemotherapy and auto-transplantation.

There have been a few reports on the coexistence of colon cancer and MM; therefore, MM is often misdiagnosed as colon adenocarcinoma metastasis.

Retrospectively, certain points in this case require further consideration. First, the colon cancer in this patient had been diagnosed and resected at an early stage, which reduced the 


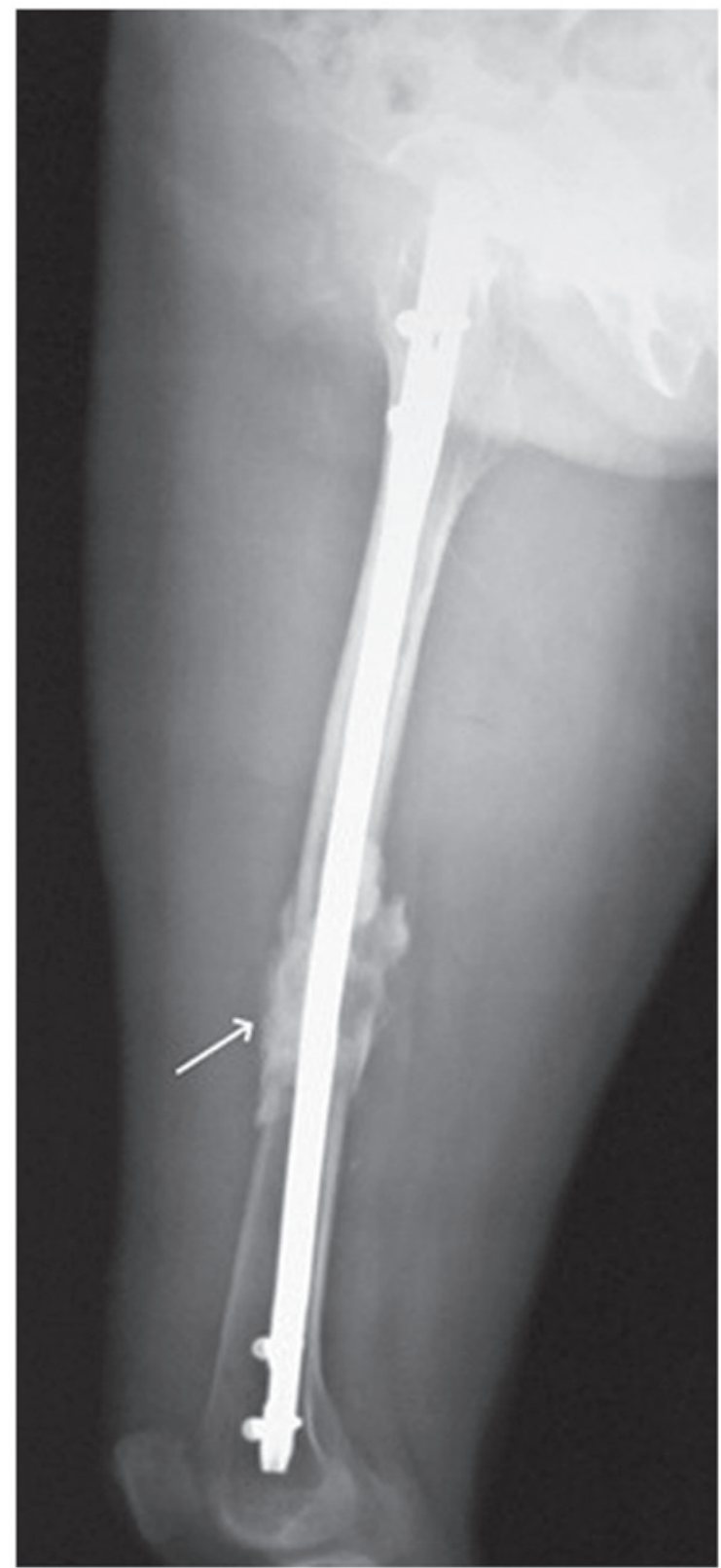

Figure 3. Roentgenogram following curettage of the tumor, filling of the bone defect with bone cement and fixing with intramedullary nails.

possibility of metastasis. Second, the most common sites of osseous metastases from colon cancer are the vertebrae, skull, pelvic bones, proximal end of the femur and humerus (2). However, the lesion in this case was in the distal diaphysis of the femur, which is unusual. Third, the colon cancer marker levels were normal and the roentgenogram revealed osteolytic lesions of the femur without periosteal reaction, which resembles MM, while the osteolytic lesions of bone metastasis are usually accompanied by periosteal reaction.

To further investigate the possibility of metastatic disease, combined ${ }^{18} \mathrm{~F}-\mathrm{FDG}$ PET/CT imaging was performed. By directly imaging the increased metabolic activity of the abnormal marrow $(12,13)$, early lesions in the humerus bilaterally and the right scapula were detected, which were missed on radiography and bone scan. However, focal uptake on PET/CT imaging is only a sign of active disease, which cannot distinguish MM from colon cancer metastasis.

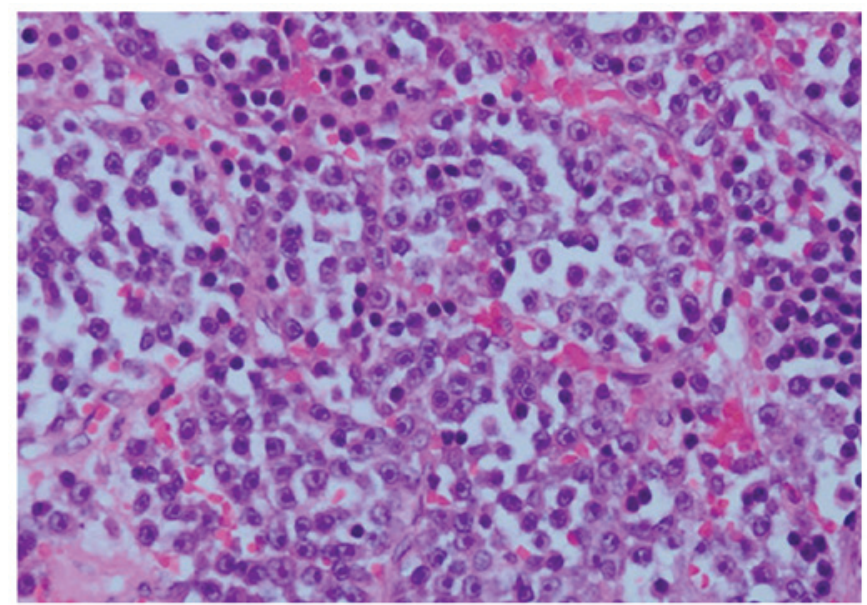

Figure 4. Histopathological examination of the right femur lesion revealed atypical plasma cells (hematoxylin and eosin staining; magnification, x400).

Therefore, new bone lesions in cancer patients should be diagnosed carefully, taking into consideration that MM may respond to timely and effective treatment.

The prognosis of colon cancer metastatic to the bone is poor. Bone metastases are generally treated by systemic chemotherapy, local radiotherapy and palliative surgery to improve the quality of life. By contrast, the outcome of MM patients has significantly improved over the last 2 decades, first through the introduction of high-dose therapy followed by autologous stem cell transplantation (ASCT) and, more recently, due to the use of proteasome inhibitors (bortezomib and carfilzomib) and immunomodulatory agents (thalidomide, lenalidomide and pomalidomide). Novel drugs are also emerging, including second- and third-generation proteasome inhibitors and immunomodulators, monoclonal antibodies, histone deacetylase inhibitors and kinesin spindle protein inhibitors.

There is an ongoing 'cure vs. control' debate on whether MM should be treated with an aggressive multidrug strategy targeting complete response (CR), or with a sequential disease control approach, in which $\mathrm{CR}$, although desirable, is not pursued as a specific treatment goal (14). Based on recent data, high-risk patients require a CR for long-term survival and, hence, clearly require an aggressive strategy (15). On the other hand, standard-risk patients exhibit similar overall survival, regardless of whether $\mathrm{CR}$ is achieved; therefore, they have the option of pursuing either an aggressive or a more conservative sequential approach. Typically, patients are treated with 2-4 cycles of induction therapy prior to harvesting stem cells (16). After the harvest, patients may either undergo frontline ASCT or resume induction therapy, delaying ASCT until the first relapse. In patients with newly diagnosed MM who are considered ineligible for ASCT due to age or other comorbidities, the main options at present are melphalan-based combination therapies (17). With melphalan-based therapy, patients are usually treated for a fixed duration of time (9-18 months) and then observed. ASCT may improve CR rates in MM (18-21). Of note, 3 randomized trials demonstrated that survival is similar whether ASCT is performed early (immediately following 4 cycles of induction therapy) or delayed (at the time of relapse as salvage therapy) (22-24). 
In conclusion, when patients with a history of colon cancer develop an osteolytic lesion, MM should be considered, and bone marrow aspiration and biopsy is mandatory. Once MM is diagnosed, standard chemotherapeutic agents should be administered immediately. The results of the present study may have implications in the treatment of similar patients.

\section{References}

1. Kanthan R, Loewy J and Kanthan SC: Skeletal metastases in colorectal carcinomas: A Saskatchewan profile. Dis Colon Rectum 42: 1592-1597, 1999.

2. Besbeas S and Stearns MW Jr: Osseous metastases from carcinomas of the colon and rectum. Dis Colon Rectum 21: 266-268, 1978.

3. Chang CY, Peng YJ, Shen DH, Huang WS and Cherng SC: Detection of multiple myeloma by PET/CT in a patient with colon cancer. Clin Nucl Med 33: 367-370, 2008.

4. Yermiahu T, Peiser J, Benharroch D and Ovnat A: Aggressive behavior of carcinoma of the colon associated with nonsecreting plasma cell myeloma. A case report. J Clin Gastroenterol 11: 565-567, 1989.

5. Zaidi AA and Vesole DH: Multiple myeloma: An old disease with new hope for the future. CA Cancer J Clin 51: 273-285, 2001.

6. Tan D, Chng WJ, Chou T, Nawarawong W, Hwang SY, Chim CS, Chen W, Durie BG and Lee JH: Management of multiple myeloma in Asia: Resource-stratified guidelines. Lancet Oncol 14: e571-e581, 2013.

7. Kyle RA: Multiple myeloma. An update on diagnosis and management. Acta Oncol 29: 1-8, 1990.

8. Kyle RA and Rajkumar SV: Criteria for diagnosis, staging, risk stratification and response assessment of multiple myeloma. Leukemia 23: 3-9, 2009.

9. Kyle RA, Therneau TM, Rajkumar SV, Remstein ED, Offord JR, Larson DR, Plevak MF and Melton LJ III: Long-term follow-up of IgM monoclonal gammopathy of undetermined significance. Blood 102: 3759-3764, 2003.

10. Snozek CLH, Katzmann JA, Kyle RA, Dispenzieri A, Larson DR, Therneau TM, Melton LJ III, Kumar S, Greipp PR, Clark RJ, et al: Prognostic value of the serum free light chain ratio in newly diagnosed myeloma: Proposed incorporation into the international staging system. Leukemia 22: 1933-1937, 2008.

11. International Myeloma Working Group: Criteria for the classification of monoclonal gammopathies, multiple myeloma and related disorders: A report of the International Myeloma Working Group. Br J Haematol 121: 749-757, 2003.

12. Jadvar H and Conti PS: Diagnostic utility of FDG PET in multiple myeloma. Skeletal Radiol 31: 690-694, 2002.

13. Schirrmeister H, Bommer M, Buck AK, Müller S, Messer P, Bunjes D, Döhner H, Bergmann L and Reske SN: Initial results in the assessment of multiple myeloma using ${ }^{18} \mathrm{~F}-\mathrm{FDG}$ PET. Eur J Nucl Med Mol Imaging 29: 361-366, 2002.
14. Rajkumar SV: Treatment of myeloma: Cure vs. control. Mayo Clin Proc 83: 1142-1145, 2008.

15. Haessler J, Shaughnessy JD Jr, Zhan F, Crowley J, Epstein J, van Rhee F, Anaissie E, Pineda-Roman M, Zangari M, Hollmig K, et al: Benefit of complete response in multiple myeloma limited to high-risk subgroup identified by gene expression profiling. Clin Cancer Res 13: 7073-7079, 2007.

16. Kumar S, Giralt S, Stadtmauer EA, Harousseau JL, Palumbo A, Bensinger W, Comenzo RL, Lentzsch S, Munshi N, Niesvizky R, et al; International Myeloma Working Group: Mobilization in myeloma revisited: IMWG consensus perspectives on stem cell collection following initial therapy with thalidomide-, lenalidomide-, or bortezomib-containing regimens. Blood 114: 1729-1735, 2009.

17. Palumbo A, Sezer O, Kyle R, Miguel JS, Orlowski RZ, Moreau P, Niesvizky R, Morgan G, Comenzo R, Sonneveld P, et al; IMWG: International Myeloma Working Group guidelines for the management of multiple myeloma patients ineligible for standard high-dose chemotherapy with autologous stem cell transplantation. Leukemia 23: 1716-1730, 2009.

18. Attal M, Harousseau JL, Stoppa AM, Sotto JJ, Fuzibet JG, Rossi JF, Casassus P, Maisonneuve H, Facon T, Ifrah N, et al: A prospective, randomized trial of autologous bone marrow transplantation and chemotherapy in multiple myeloma. Intergroupe Français du Myélome. N Engl J Med 335: 91-97, 1996.

19. Child JA, Morgan GJ, Davies FE, Owen RG, Bell SE, Hawkins K, Brown J, Drayson MT and Selby PJ; Medical Research Council Adult Leukaemia Working Party: High-dose chemotherapy with hematopoietic stem-cell rescue for multiple myeloma. N Engl J Med 348: 1875-1883, 2003.

20. Blade J, Vesole DH and Gertz M: Transplantation for multiple myeloma: Who, when, how often? Blood 102: 3469-3477, 2003.

21. Kumar A, Loughran T, Alsina M, Durie BG and Djulbegovic B: Management of multiple myeloma: A systematic review and critical appraisal of published studies. Lancet Oncol 4: 293-304, 2003.

22. Fermand JP, Ravaud P, Chevret S, Divine M, Leblond V, Belanger C, Macro M, Pertuiset E, Dreyfus F, Mariette X, et al: High-dose therapy and autologous peripheral blood stem cell transplantation in multiple myeloma: Up-front or rescue treatment? Results of a multicenter sequential randomized clinical trial. Blood 92: 3131-3136, 1998.

23. Facon T, Mary JY, Harousseau JL, et al: Front-line or rescue autologous bone marrow transplantation (ABMT) following a first course of high dose melphalan (HDM) in multiple myeloma (MM). Preliminary results of a prospective randomized trial (CIAM) protocol. Blood 88 (Suppl 1): 685a, 1996.

24. Barlogie B, Kyle R, Anderson K, et al: Comparable survival in multiple myeloma (MM) with high dose therapy (HDT) employing MEL $140 \mathrm{mg} / \mathrm{m}^{2}$ TBI 12 Gy autotransplants vs. standard dose therapy with VBMCP and no benefit from interferon (IFN) maintenance: Results of intergroup Trial S9321. Blood 102: 42a, 2003. 